\title{
Finding a solution of split null point of the sum of mono- tone operators without prior knowledge of operator norms in Hilbert spaces
}

\author{
Montira Suwannaprapa ${ }^{\mathrm{a}}$, Narin Petrot ${ }^{\mathrm{a}, \mathrm{b}, *}$ \\ ${ }^{a}$ Department of Mathematics, Faculty of Science, Naresuan University, Phitsanulok 65000, Thailand. \\ ${ }^{b}$ Centre of Excellence in Nonlinear Analysis and Optimizations, Faculty of Science, Naresuan University, Phitsanulok 65000, Thailand. \\ Communicated by Y. J. Cho
}

\begin{abstract}
In this paper, we consider the split monotone variational inclusion problem in Hilbert spaces. By assuming the existence of solutions, we introduce an iterative algorithm, in which the stepsizes does not need any prior information about the operator norm, and show its convergence theorem. Some applications and numerical experiments of the considered problem are also discussed.
\end{abstract}

Keywords: Split monotone variational inclusion problem, maximal monotone operator, inverse strongly monotone operator, convergence theorems.

2010 MSC: 49J53, 26A18.

(C)2018 All rights reserved.

\section{Introduction}

Variational inclusion problems (VIP) are being used as mathematical programming models to study a large number of optimization problems arising in finance, economics, network, transportation, and engineering science. For a Hilbert space $H$, the formal form of VIP is a problem of finding $x^{*} \in H$ such that

$$
0 \in \mathrm{B} x^{*}
$$

where $\mathrm{B}: \mathrm{H} \rightarrow 2^{\mathrm{H}}$ is a set-valued operator. If $\mathrm{B}$ is a maximal monotone operator, the elements in the solution set of the problem (1.1) are called the zeros of maximal monotone operator. This problem was introduced by Martinet [24], and later it has been studied by many authors. It is well-known that the popular iteration method that was used for solving the problem (1.1) is the following proximal point algorithm: for a given $x_{1} \in \mathrm{H}$,

$$
x_{\mathrm{n}+1}=\mathrm{J}_{\lambda_{\mathrm{n}}}^{\mathrm{B}} \mathrm{x}_{\mathrm{n}}, \quad \forall \mathrm{n} \in \mathbb{N},
$$

where $\left\{\lambda_{n}\right\} \subset(0, \infty)$ and $J_{\lambda_{n}}^{B}=\left(I+\lambda_{n} B\right)^{-1}$ is the resolvent of the considered maximal monotone operator

\footnotetext{
${ }^{*}$ Corresponding author

Email addresses: montira.sw@gmail .com (Montira Suwannaprapa), narinp@nu.ac.th (Narin Petrot)

doi: 10.22436/jnsa.011.05.09
}

Received: 2017-10-16 Revised: 2017-12-20 Accepted: 2018-03-02 
B corresponding to $\lambda_{n}$, see also $[5,17,22,37,39]$ for more details.

On the other hand, split feasibility problem (SFP), which was first introduced by Censor and Elfving [10], have been appeared in various fields of science and technology, such as in signal processing, medical image reconstruction, and intensity-modulated radiation therapy, for more information, see [6, 9] and the references therein. The SFP is the problem of finding a point

$$
x^{*} \in \mathrm{C} \quad \text { such that } \mathrm{L} x^{*} \in \mathrm{Q},
$$

where $C$ and $Q$ are nonempty closed convex subsets of $\mathbb{R}^{n}$ and $\mathbb{R}^{m}$, respectively, and $L$ is an $m \times n$ matrix. Later on, Byrne [6] suggested a following CQ algorithm: for arbitrary $x_{1} \in \mathbb{R}^{n}$,

$$
x_{n+1}=P_{C}\left(x_{n}+\gamma L^{t}\left(P_{Q}-I\right) L x_{n}\right), \quad \forall n \in \mathbb{N},
$$

where $\gamma \in\left(0,2 /\|\mathrm{L}\|^{2}\right), \mathrm{L}^{\mathrm{t}}$ is the transpose of the considered matrix $\mathrm{L}$, and $\mathrm{P}_{\mathrm{C}}$ and $\mathrm{P}_{\mathrm{Q}}$ are the metric projections onto $\mathrm{C}$ and $\mathrm{Q}$, respectively. Subsequently, by considering the CQ algorithm (1.2), López et al. [21] suggested to use the stepsizes $\gamma_{n}$ without the norm of operator $L$,

$$
\gamma_{n}=\frac{\rho_{n}\left\|\left(I-P_{Q}\right) L x_{n}\right\|^{2}}{2\left\|L^{*}\left(I-P_{Q}\right) L x_{n}\right\|^{2}}
$$

where $0<\rho_{n}<4$ and $\mathrm{L}^{*}\left(\mathrm{I}-\mathrm{P}_{\mathrm{Q}}\right) \mathrm{L} x_{\mathrm{n}} \neq 0$, and proved weakly convergence theorem. López et al. [21] pointed out that, the higher dimensions of L may be hard to compute the operator norm and it may effect to the computing in the iteration process, for example the CPU time, and the algorithm with stepsizes (1.3) gives a faster results.

In 2012, by combining the concepts of VIP and SFP, Byrne et al. [7] introduced and studied the following split null point problem (SNPP): find $x^{*} \in \mathrm{H}_{1}$ such that

$$
0 \in \mathrm{B}_{1}\left(x^{*}\right) \text { and } 0 \in \mathrm{B}_{2}\left(\mathrm{Lx}^{*}\right),
$$

where $\mathrm{B}_{i}: \mathrm{H}_{i} \rightarrow 2^{\mathrm{H}_{i}}, \mathrm{i}=1,2$ are maximal monotone operators. Byrne et al. [7] considered the following iterative algorithm: for $\lambda>0$ and an arbitrary $x_{1} \in H_{1}$,

$$
x_{n+1}=J_{\lambda}^{B_{1}}\left(x_{n}-\gamma L^{*}\left(I-J_{\lambda}^{B_{2}}\right) L x_{n}\right), \quad \forall n \in \mathbb{N},
$$

where $\gamma \in\left(0,2 /\|\mathrm{L}\|^{2}\right)$, and proved that $\left\{x_{n}\right\}$ converges weakly to a point $x^{*}$ in the solution set of problem (1.4).

Now, let us go back to the VIP problem (1.1). A type of generalization of problem (1.1) is a problem to find a point $x^{*} \in H$ such that

$$
0 \in A x^{*}+B x^{*}
$$

where $\mathrm{A}: \mathrm{H} \rightarrow \mathrm{H}$ is a single-valued operator and $\mathrm{B}: \mathrm{H} \rightarrow 2^{\mathrm{H}}$ is a set-valued operator. It is well known that, there are many kinds of real world problems those rise in the form of problem (1.5), see $[4,28,29,36]$ for example and the references therein. In the case that $A: H \rightarrow H$ is a single-valued monotone operator and $\mathrm{B}: \mathrm{H} \rightarrow 2^{\mathrm{H}}$ is a set-valued monotone operator, the elements in the solution set of the problem (1.5) are called the zeros of the sum of monotone operators. Note that $0 \in A x^{*}+B x^{*}$ is equivalent to $J_{\lambda}^{B}(I-\lambda A) x^{*}=x^{*}$, when $\lambda>0$, see [3]. It is worth to note that, there are many authors considered the methods for finding zeros of the operators which are also a solution to another problems, see $[12-15,30,32]$ for example.

In 2011, Moudafi [27] introduced the following split monotone variational inclusion (SMVI): let $\mathrm{H}_{1}$ and $H_{2}$ be two real Hilbert spaces, $A_{i}: H_{i} \rightarrow H_{i}, i=1,2$ be single-valued operators, $B_{i}: H_{i} \rightarrow 2^{H_{i}}$, $i=1,2$ be set-valued operators, and $L: H_{1} \rightarrow H_{2}$ be a bounded linear operator. The SMVI is the following problem:

$$
\text { find } x^{*} \in H_{1} \text { such that } 0 \in\left(A_{1}+B_{1}\right) x^{*} \text {, }
$$


and such that

$$
\text { Lx } x^{*} \in \mathrm{H}_{2} \text { solves } 0 \in\left(\mathrm{A}_{2}+\mathrm{B}_{2}\right) \mathrm{Lx} \text {. }
$$

We will denote the solution set of the problem (1.6) by $\Gamma$. Moudafi [27] proposed the following iterative algorithm: let $A_{i}: H_{i} \rightarrow H_{i}, i=1,2$ be $\alpha_{i}$-ism and $\alpha:=\min \left\{\alpha_{i}\right\}, i=1,2, U:=J_{\lambda}^{B_{1}}\left(I-\lambda A_{1}\right)$ and $\mathrm{T}:=\mathrm{J}_{\lambda}^{\mathrm{B}_{2}}\left(\mathrm{I}-\lambda \mathrm{A}_{2}\right)$ with $\lambda \in(0,2 \alpha)$. For arbitrary $x_{1} \in \mathrm{H}_{1}$,

$$
x_{n+1}=U\left(x_{n}+\gamma L^{*}(T-I) L x_{n}\right), \quad n \in \mathbb{N},
$$

where $\gamma \in\left(0,1 /\|\mathrm{L}\|^{2}\right)$, and proved that any sequence $\left\{x_{n}\right\}$ generated by the algorithm (1.7) converges weakly to $x^{*} \in \Gamma$.

In this paper, motivated and inspired by above literatures, we are going to consider a problem (1.6) and suggest the following algorithm: for an arbitrary initial $x_{1} \in \mathrm{H}_{1}$,

$$
\left\{\begin{array}{l}
u_{n}=x_{n}-\gamma_{n} L^{*}\left(I-J_{\lambda}^{B_{2}}\left(I-\lambda A_{2}\right)\right) L x_{n}, \\
x_{n+1}=\alpha_{n} \nabla h\left(u_{n}\right)+\left(1-\alpha_{n}\right) J_{\lambda}^{B_{1}}\left(I-\lambda A_{1}\right) u_{n}, \quad \forall n \in \mathbb{N},
\end{array}\right.
$$

where $h: H_{1} \rightarrow \mathbb{R}$ is a continuous differentiable function, $\left\{\alpha_{n}\right\} \subset(0,1)$ and choose the stepsizes sequence $\left\{\gamma_{n}\right\}$ which does not need any prior information about the operator norm. In our main results, we show that the constructed sequence $\left\{x_{n}\right\}$ converges strongly to a point $\bar{x} \in \Gamma$, where $\bar{x}=P_{\Gamma} \nabla h(\bar{x})$, which is the optimality condition for the minimization problem

$$
\min _{x \in \Gamma} \frac{1}{2}\|x\|^{2}-h(x)
$$

\section{Preliminaries}

Throughout this paper, we denote by $\mathbb{N}$ for the set of positive integers, and $\mathbb{R}$ for the set of real numbers. Let $\mathrm{H}$ be a real Hilbert space with the inner product $\langle\cdot, \cdot\rangle$ and the norm $\|\cdot\|$, respectively. When $\left\{x_{n}\right\}$ is a sequence in $H$, we denote the strong convergence and weak convergence of $\left\{x_{n}\right\}$ to $x$ in $H$ by $x_{n} \rightarrow x$ and $x_{n} \rightarrow x$, respectively.

Let $\mathrm{T}: \mathrm{H} \rightarrow \mathrm{H}$ be a mapping. We say that $\mathrm{T}$ is a Lipschitz mapping if there exists $\mathrm{L} \geqslant 0$ such that

$$
\|\mathrm{T} x-\mathrm{T} y\| \leqslant \mathrm{L}\|x-y\|, \quad \forall x, y \in H .
$$

The number $L$, associated with $T$, is called a Lipschitz constant. If $L \in[0,1)$, we say that $T$ is a contraction mapping, and $\mathrm{T}$ is a nonexpansive mapping if $\mathrm{L}=1$.

We say that $T$ is strongly nonexpansive if $T$ is nonexpansive and for all bounded sequences $\left\{x_{n}\right\},\left\{y_{n}\right\}$ in $H$, the condition $\lim _{n \rightarrow \infty}\left(\left\|x_{n}-y_{n}\right\|-\left\|T x_{n}-T y_{n}\right\|\right)=0$ implies $\lim _{n \rightarrow \infty}\left\|\left(x_{n}-y_{n}\right)-\left(T x_{n}-T y_{n}\right)\right\|=0$, see [8].

We will say that $T$ is firmly nonexpansive if

$$
\langle\mathrm{T} x-\mathrm{T} y, x-\mathrm{y}\rangle \geqslant\|\mathrm{T} x-\mathrm{T} y\|^{2}, \quad \forall x, y \in H .
$$

The set of fixed points of a self mapping $T$ will be denoted by $F(T)$, that is $F(T)=\{x \in H: T x=x\}$. It is well known that if $T$ is nonexpansive, then $F(T)$ is closed and convex.

A mapping $\mathrm{T}: \mathrm{H} \rightarrow \mathrm{H}$ is said to be an averaged mapping if it can be written as the average of the identity I and a nonexpansive mapping, that is,

$$
\mathrm{T}=(1-\alpha) \mathrm{I}+\alpha \mathrm{S},
$$

where $\alpha \in(0,1)$ and $S: H \rightarrow H$ is a nonexpansive mapping, see [1]. More precisely, when (2.1) holds, we say that $\mathrm{T}$ is $\alpha$-averaged. It should be observed that firmly nonexpansive mappings are $\frac{1}{2}$-averaged mappings. We also note that, averaged mappings are strongly nonexpansive, see [8]. 
Let $A: H \rightarrow H$ be a single-valued mapping. For a positive real number $\beta$, we will say that $A$ is $\beta$-inverse strongly monotone $(\beta$-ism) if

$$
\langle A x-A y, x-y\rangle \geqslant \beta\|A x-A y\|^{2}, \quad \forall x, y \in H .
$$

The classes of inverse strongly monotone mappings have been studied by many authors, see [4, 38].

We now collect some important properties, that are needed in this work.

Lemma 2.1 ([4, 38]). We have

(i) the composite of finitely many averaged mappings is averaged. In particular, if $\mathrm{T}_{i}$ is $\alpha_{i}$-averaged, where $\alpha_{i} \in(0,1)$ for $i=1,2$, then the composite $T_{1} T_{2}$ is $\alpha$-averaged, where $\alpha=\alpha_{1}+\alpha_{2}-\alpha_{1} \alpha_{2}$;

(ii) if $A$ is $\beta$-ism and $\lambda \in(0, \beta]$, then $T:=I-\lambda A$ is firmly nonexpansive;

(iii) a mapping $\mathrm{T}: \mathrm{H} \rightarrow \mathrm{H}$ is nonexpansive if and only if $\mathrm{I}-\mathrm{T}$ is $\frac{1}{2}$-ism;

(iv) $\mathrm{T}$ is averaged if and only if the complement $\mathrm{I}-\mathrm{T}$ is $\beta$-ism for some $\beta>\frac{1}{2}$. Indeed, for $\alpha \in(0,1), \mathrm{T}$ is $\alpha$-averaged if and only if $\mathrm{I}-\mathrm{T}$ is $\frac{1}{2 \alpha}-i s m$.

Let $\mathrm{B}: \mathrm{H} \rightarrow 2^{\mathrm{H}}$ be a set-valued mapping. The effective domain of $\mathrm{B}$ is denoted by $D(B)$, that is, $D(B)=\{x \in H: B x \neq \emptyset\}$. Recall that $B$ is said to be monotone if

$$
\langle x-y, u-v\rangle \geqslant 0, \quad \forall x, y \in D(B), u \in B x, v \in B y .
$$

A monotone mapping B is said to be maximal if its graph is not properly contained in the graph of any other monotone operator. To a maximal monotone operator $\mathrm{B}: \mathrm{H} \rightarrow 2^{\mathrm{H}}$ and $\lambda>0$, its resolvent $\mathrm{J}_{\lambda}^{\mathrm{B}}$ is defined by

$$
\mathrm{J}_{\lambda}^{\mathrm{B}}:=(\mathrm{I}+\lambda \mathrm{B})^{-1}: \mathrm{H} \rightarrow \mathrm{D}(\mathrm{B}) .
$$

It is well known that if $B$ is a maximal monotone operator and $\lambda$ is a positive number, then the resolvent $J_{\lambda}^{B}$ is a single-valued and firmly nonexpansive, and $F\left(J_{\lambda}^{B}\right)=B^{-1} 0 \equiv\{x \in H: 0 \in B x\}, \quad \forall \lambda>0$, see [33, 35].

The following fundamental results and inequalities are needed in our proof.

Let $C$ be a nonempty closed convex subset of $H$. For every point $x \in H$, there exists a unique nearest point in $\mathrm{C}$, denoted by $\mathrm{P}_{\mathrm{C}} \mathrm{x}$, such that

$$
\left\|x-P_{C} x\right\| \leqslant\|x-y\|, \quad \forall y \in C .
$$

$P_{C}$ is called a metric projection of $H$ onto $C$, see [34]. The following property of $P_{C}$ is well known and useful:

$$
\left\langle x-P_{C} x, y-P_{C} x\right\rangle \leqslant 0, \quad \forall x \in H, y \in C .
$$

For each $x, y \in H$ and $\lambda \in(0,1)$, we know that

$$
\|x+y\|^{2} \leqslant\|x\|^{2}+2\langle y, x+y\rangle
$$

and

$$
\|\lambda x+(1-\lambda) y\|^{2}=\lambda\|x\|^{2}+(1-\lambda)\|y\|^{2}-\lambda(1-\lambda)\|x-y\|^{2},
$$

see [4].

We also use the following lemma for proving the main results.

Lemma 2.2 ([20, 37]). Let $\left\{a_{n}\right\}$ be a sequence of nonnegative real numbers satisfying the following relation:

$$
a_{n+1} \leqslant\left(1-\alpha_{n}\right) a_{n}+\alpha_{n} \sigma_{n}+\delta_{n}, \quad \forall n \in \mathbb{N},
$$

where $\left\{\alpha_{n}\right\},\left\{\sigma_{n}\right\}$, and $\left\{\delta_{n}\right\}$ are sequences of real numbers satisfying

(i) $\left\{\alpha_{n}\right\} \subset[0,1], \sum_{i=1}^{\infty} \alpha_{n}=\infty$;

(ii) $\limsup _{n \rightarrow \infty} \sigma_{n} \leqslant 0$;

(iii) $\delta_{n} \geqslant 0, \sum_{i=1}^{\infty} \delta_{n}<\infty$.

Then, $\mathrm{a}_{\mathrm{n}} \rightarrow 0$ as $\mathrm{n} \rightarrow \infty$. 


\section{Main results}

We start by introducing assumptions and an iterative algorithm in which the stepsizes does not depend on the norm of operator L. We will use the following assumptions in order to provide the convergence theorems.

(A1) $A_{i}: H_{i} \rightarrow H_{i}, i=1,2$ are inverse strongly monotone operators;

(A2) $B_{i}: H_{i} \rightarrow 2^{H_{i}}, i=1,2$ are maximal monotone operators, such that $\left(A_{i}+B_{i}\right)^{-1} 0 \neq \emptyset$;

(A3) $J_{\lambda}^{B_{1}}$ and $J_{\lambda}^{B_{2}}$, for $\lambda>0$, are resolvents of $B_{1}$ and $B_{2}$, respectively;

(A4) $\mathrm{L}: \mathrm{H}_{1} \rightarrow \mathrm{H}_{2}$ is a bounded linear operator, such that $\mathrm{L} \neq 0$;

(A5) $h: \mathrm{H}_{1} \rightarrow \mathbb{R}$ is a differentiable function, such that $\nabla h$ is a contraction with coefficient $k \in(0,1)$.

Algorithm 3.1. Let $\left\{\alpha_{n}\right\} \subset(0,1)$ and an initial $x_{1} \in \mathrm{H}_{1}$ be arbitrary, define

$$
\left\{\begin{array}{l}
u_{n}=x_{n}-\gamma_{n} L^{*}\left(I-J_{\lambda}^{B_{2}}\left(I-\lambda A_{2}\right)\right) L x_{n}, \\
x_{n+1}=\alpha_{n} \nabla h\left(u_{n}\right)+\left(1-\alpha_{n}\right) J_{\lambda}^{B_{1}}\left(I-\lambda A_{1}\right) u_{n}, \quad \forall n \in \mathbb{N}
\end{array}\right.
$$

where the stepsizes sequence $\left\{\gamma_{n}\right\}$ depends on $\rho_{n} \in[a, b] \subset(0,1)$ by

$$
\gamma_{n}= \begin{cases}\rho_{n} \frac{\left\|\left(I-J_{\lambda}^{B_{2}}\left(I-\lambda A_{2}\right)\right) L x_{n}\right\|^{2}}{\left\|L^{*}\left(I-J_{\lambda}^{B_{2}}\left(I-\lambda A_{2}\right)\right) L x_{n}\right\|^{2}}, & \text { if } \quad L^{*}\left(I-J_{\lambda}^{B_{2}}\left(I-\lambda A_{2}\right)\right) L x_{n} \neq 0, \\ \gamma, & \text { if otherwise, }\end{cases}
$$

where $\gamma$ is any nonnegative value.

Remark 3.2. It is worth to point out that the Algorithm 3.1 is a type of viscosity algorithm, see [23, 25] for more information.

Remark 3.3. The stepsizes $\left\{\gamma_{n}\right\}$ is bounded. Indeed, for each $n \in \mathbb{N}$,

$$
\left\|L^{*}\left(I-J_{\lambda}^{B_{2}}\left(I-\lambda A_{2}\right)\right) L x_{n}\right\| \leqslant\left\|L^{*}\right\|\left\|\left(I-J_{\lambda}^{B_{2}}\left(I-\lambda A_{2}\right)\right) L x_{n}\right\|,
$$

which implies

$$
\frac{\left\|\left(I-J_{\lambda}^{B_{2}}\left(I-\lambda A_{2}\right)\right) L x_{n}\right\|^{2}}{\left\|L^{*}\left(I-J_{\lambda}^{B_{2}}\left(I-\lambda A_{2}\right)\right) L x_{n}\right\|^{2}} \geqslant \frac{1}{\left\|L^{*}\right\|^{2}} .
$$

Let $w_{n}=\frac{\left\|\left(I-J_{\lambda}^{B_{2}}\left(I-\lambda A_{2}\right)\right) L x_{n}\right\|^{2}}{\left\|L^{*}\left(I-J_{\lambda}^{B_{2}}\left(I-\lambda A_{2}\right)\right) L x_{n}\right\|^{2}}$ and $L^{*}\left(I-J_{\lambda}^{B_{2}}\left(I-\lambda A_{2}\right)\right) L x_{n} \neq 0$ for each $n \in \mathbb{N}$. It follows from the definition of $\left\{\gamma_{n}\right\}$, we can see that $\sup \gamma_{n}<\inf w_{n}<\infty$. This means $\left\{\gamma_{n}\right\}$ is bounded.

Lemma 3.4. Let $\mathrm{H}_{1}$ and $\mathrm{H}_{2}$ be two real Hilbert spaces. Let $\left\{x_{\mathrm{n}}\right\}$ be generated by Algorithm 3.1. Suppose that the assumptions (A1)-(A5) hold and $\Gamma \neq \emptyset$. Then, the sequences $\left\{x_{n}\right\}$ and $\left\{u_{n}\right\}$ are bounded.

Proof. Let $z \in \Gamma$. It follows that $z=J_{\lambda}^{B_{1}}\left(I-\lambda A_{1}\right) z$ and $L z=J_{\lambda}^{B_{2}}\left(I-\lambda A_{2}\right) L z$. We note that $J_{\lambda}^{B_{2}}$ is $\frac{1}{2}$-averaged. Since $A_{2}$ is inverse strongly operator, say $\beta$-ism, so by Lemma 2.1 (ii), for each $\lambda \in(0, \beta)$, we know that $\left(I-\lambda A_{2}\right)$ is $\frac{1}{2}$-averaged. Thus, by Lemma $2.1(i)$, we have $J_{\lambda}^{B_{2}}\left(I-\lambda A_{2}\right)$ is $\frac{3}{4}$-averaged. Subsequently, by Lemma 2.1 (iv), we have $I-J_{\lambda}^{B_{2}}\left(I-\lambda A_{2}\right)$ is $\frac{2}{3}$-ism. It follows that, for each $n \in \mathbb{N}$,

$$
\left\langle\left(I-J_{\lambda}^{B_{2}}\left(I-\lambda A_{2}\right)\right) L x_{n}-\left(I-J_{\lambda}^{B_{2}}\left(I-\lambda A_{2}\right)\right) L z, L x_{n}-L z\right\rangle \geqslant \frac{2}{3}\left\|\left(I-J_{\lambda}^{B_{2}}\left(I-\lambda A_{2}\right)\right) L x_{n}-\left(I-J_{\lambda}^{B_{2}}\left(I-\lambda A_{2}\right)\right) L z\right\|^{2} .
$$

Since $L z=J_{\lambda}^{B_{2}}\left(I-\lambda A_{2}\right) L z$, the above inequality is reduced to

$$
\left\langle\left(\mathrm{I}-\mathrm{J}_{\lambda}^{\mathrm{B}_{2}}\left(\mathrm{I}-\lambda \mathrm{A}_{2}\right)\right) \mathrm{L} x_{\mathrm{n}}, \mathrm{L} x_{\mathrm{n}}-\mathrm{L} z\right\rangle \geqslant \frac{2}{3}\left\|\left(\mathrm{I}-\mathrm{J}_{\lambda}^{\mathrm{B}_{2}}\left(\mathrm{I}-\lambda \mathrm{A}_{2}\right)\right) \mathrm{L} x_{\mathrm{n}}\right\|^{2}
$$


for each $n \in \mathbb{N}$. By using (3.1), we see that

$$
\begin{aligned}
\left\|u_{n}-z\right\|^{2} & =\left\|x_{n}-\gamma_{n} L^{*}\left(I-J_{\lambda}^{B_{2}}\left(I-\lambda A_{2}\right)\right) L x_{n}-z\right\|^{2} \\
& =\left\|\left(x_{n}-z\right)-\gamma_{n} L^{*}\left(I-J_{\lambda}^{B_{2}}\left(I-\lambda A_{2}\right)\right) L x_{n}\right\|^{2} \\
& =\left\|x_{n}-z\right\|^{2}-2 \gamma_{n}\left\langle x_{n}-z, L^{*}\left(I-J_{\lambda}^{B_{2}}\left(I-\lambda A_{2}\right)\right) L x_{n}\right\rangle+\gamma_{n}^{2}\left\|L^{*}\left(I-J_{\lambda}^{B_{2}}\left(I-\lambda A_{2}\right)\right) L x_{n}\right\|^{2} \\
& =\left\|x_{n}-z\right\|^{2}-2 \gamma_{n}\left\langle L x_{n}-L z,\left(I-J_{\lambda}^{B_{2}}\left(I-\lambda A_{2}\right)\right) L x_{n}\right\rangle+\gamma_{n}^{2}\left\|L^{*}\left(I-J_{\lambda}^{B_{2}}\left(I-\lambda A_{2}\right)\right) L x_{n}\right\|^{2} \\
& \leqslant\left\|x_{n}-z\right\|^{2}-\frac{4 \gamma_{n}}{3}\left\|\left(I-J_{\lambda}^{B_{2}}\left(I-\lambda A_{2}\right)\right) L x_{n}\right\|^{2}+\gamma_{n}^{2}\left\|L^{*}\left(I-J_{\lambda}^{B_{2}}\left(I-\lambda A_{2}\right)\right) L x_{n}\right\|^{2} \\
& \leqslant\left\|x_{n}-z\right\|^{2}-\gamma_{n}\left\|\left(I-J_{\lambda}^{B_{2}}\left(I-\lambda A_{2}\right)\right) L x_{n}\right\|^{2}+\gamma_{n}^{2}\left\|L^{*}\left(I-J_{\lambda}^{B_{2}}\left(I-\lambda A_{2}\right)\right) L x_{n}\right\|^{2} \\
& =\left\|x_{n}-z\right\|^{2}-\gamma_{n}\left[\left\|\left(I-J_{\lambda}^{B_{2}}\left(I-\lambda A_{2}\right)\right) L x_{n}\right\|^{2}-\gamma_{n}\left\|L^{*}\left(I-J_{\lambda}^{B_{2}}\left(I-\lambda A_{2}\right)\right) L x_{n}\right\|^{2}\right]
\end{aligned}
$$

for each $n \in \mathbb{N}$. By the definition of $\gamma_{n}$, we have

$$
\left\|u_{n}-z\right\|^{2} \leqslant\left\|x_{n}-z\right\|^{2}
$$

for each $n \in \mathbb{N}$. Thus

$$
\left\|u_{n}-z\right\| \leqslant\left\|x_{n}-z\right\|
$$

for each $n \in \mathbb{N}$. Furthermore, we get

$$
\begin{aligned}
\left\|x_{n+1}-z\right\| & =\left\|\alpha_{n} \nabla h\left(u_{n}\right)+\left(1-\alpha_{n}\right) J_{\lambda}^{B_{1}}\left(I-\lambda A_{1}\right) u_{n}-z\right\| \\
& =\left\|\alpha_{n}\left(\nabla h\left(u_{n}\right)-z\right)+\left(1-\alpha_{n}\right)\left(J_{\lambda}^{B_{1}}\left(I-\lambda A_{1}\right) u_{n}-z\right)\right\| \\
& \leqslant \alpha_{n}\left\|\nabla h\left(u_{n}\right)-z\right\|+\left(1-\alpha_{n}\right)\left\|J_{\lambda}^{B_{1}}\left(I-\lambda A_{1}\right) u_{n}-z\right\| \\
& \leqslant \alpha_{n}\left\|\nabla h\left(u_{n}\right)-\nabla h(z)\right\|+\alpha_{n}\|\nabla h(z)-z\|+\left(1-\alpha_{n}\right)\left\|u_{n}-z\right\| \\
& \leqslant \alpha_{n} \kappa\left\|u_{n}-z\right\|+\alpha_{n}\|\nabla h(z)-z\|+\left(1-\alpha_{n}\right)\left\|u_{n}-z\right\| \\
& =\left(1-\alpha_{n}(1-\kappa)\right)\left\|u_{n}-z\right\|+\alpha_{n}\|\nabla h(z)-z\| \\
& \leqslant\left(1-\alpha_{n}(1-\kappa)\right)\left\|x_{n}-z\right\|+\alpha_{n}(1-\kappa)\left(\frac{\|\nabla h(z)-z\|}{1-\kappa}\right) \\
& \leqslant \max \left\{\left\|x_{n}-z\right\|, \frac{\|\nabla h(z)-z\|}{1-\kappa}\right\} \\
& \vdots \\
& \leqslant \max \left\{\left\|x_{1}-z\right\|, \frac{\|\nabla h(z)-z\|}{1-\kappa}\right\}
\end{aligned}
$$

for each $n \in \mathbb{N}$. Therefore, $\left\{\left\|x_{n}-z\right\|\right\}$ is a bounded sequence and consequently, $\left\{\left\|u_{n}-z\right\|\right\}$ is also bounded. These imply that $\left\{x_{n}\right\}$ and $\left\{u_{n}\right\}$ are bounded.

Theorem 3.5. Let $\mathrm{H}_{1}$ and $\mathrm{H}_{2}$ be two real Hilbert spaces. Let $\left\{x_{n}\right\}$ be generated by Algorithm 3.1. Suppose that the assumptions (A1)-(A5) hold, $\Gamma \neq \emptyset$ and the following control conditions are satisfied:

(i) $\lim _{n \rightarrow \infty} \alpha_{n}=0$;

(ii) $\sum_{n=1}^{\infty} \alpha_{n}=\infty$.

Then, $\left\{u_{n}\right\}$ and $\left\{x_{n}\right\}$ both converge strongly to $\bar{x} \in \Gamma$, where $\bar{x}=P_{\Gamma} \nabla h(\bar{x})$, which is the optimality condition for the minimization problem

$$
\min _{x \in \Gamma} \frac{1}{2}\|x\|^{2}-h(x)
$$


Proof. Firstly, we note that $\mathrm{P}_{\Gamma} \nabla \mathrm{h}(\cdot)$ is a contraction mapping. Let $\bar{x}$ be the unique fixed point of $\mathrm{P}_{\Gamma} \nabla h(\cdot)$, that is $\bar{x}=P_{\Gamma} \nabla h(\bar{x})$. Then, we obtain

$$
\begin{aligned}
\left\|x_{n+1}-\bar{x}\right\|^{2}= & \left\langle\alpha_{n} \nabla h\left(u_{n}\right)+\left(1-\alpha_{n}\right) J_{\lambda}^{B_{1}}\left(I-\lambda A_{1}\right) u_{n}-\bar{x}, x_{n+1}-\bar{x}\right\rangle \\
= & \alpha_{n}\left\langle\nabla h\left(u_{n}\right)-\bar{x}, x_{n+1}-\bar{x}\right\rangle+\left(1-\alpha_{n}\right)\left\langle J_{\lambda}^{B_{1}}\left(I-\lambda A_{1}\right) u_{n}-\bar{x}, x_{n+1}-\bar{x}\right\rangle \\
= & \alpha_{n}\left\langle\nabla h\left(u_{n}\right)-\nabla h(\bar{x}), x_{n+1}-\bar{x}\right\rangle+\alpha_{n}\left\langle\nabla h(\bar{x})-\bar{x}, x_{n+1}-\bar{x}\right\rangle \\
& +\left(1-\alpha_{n}\right)\left\langle J_{\lambda}^{B_{1}}\left(I-\lambda A_{1}\right) u_{n}-\bar{x}, x_{n+1}-\bar{x}\right\rangle \\
\leqslant & \alpha_{n}\left\|\nabla h\left(u_{n}\right)-\nabla h(\bar{x})\right\|\left\|x_{n+1}-\bar{x}\right\|+\left(1-\alpha_{n}\right)\left\|J_{\lambda}^{B_{1}}\left(I-\lambda A_{1}\right) u_{n}-\bar{x}\right\|\left\|x_{n+1}-\bar{x}\right\| \\
& +\alpha_{n}\left\langle\nabla h(\bar{x})-\bar{x}, x_{n+1}-\bar{x}\right\rangle \\
\leqslant & \frac{\alpha_{n}}{2}\left(\left\|\nabla h\left(u_{n}\right)-\nabla h(\bar{x})\right\|^{2}+\left\|x_{n+1}-\bar{x}\right\|^{2}\right) \\
& +\frac{1-\alpha_{n}}{2}\left(\left\|J_{\lambda}^{B_{1}}\left(I-\lambda A_{1}\right) u_{n}-\bar{x}\right\|^{2}+\left\|x_{n+1}-\bar{x}\right\|^{2}\right)+\alpha_{n}\left\langle\nabla h(\bar{x})-\bar{x}, x_{n+1}-\bar{x}\right\rangle \\
\leqslant & \frac{\alpha_{n} k^{2}}{2}\left\|u_{n}-\bar{x}\right\|^{2}+\frac{\alpha_{n}}{2}\left\|x_{n+1}-\bar{x}\right\|^{2}+\frac{1-\alpha_{n}}{2}\left\|u_{n}-\bar{x}\right\|^{2}+\frac{1-\alpha_{n}}{2}\left\|x_{n+1}-\bar{x}\right\|^{2} \\
& +\alpha_{n}\left\langle\nabla h(\bar{x})-\bar{x}, x_{n+1}-\bar{x}\right\rangle \\
\leqslant & \frac{1-\alpha_{n}\left(1-k^{2}\right)}{2}\left\|x_{n}-\bar{x}\right\|^{2}+\frac{1}{2}\left\|x_{n+1}-\bar{x}\right\|^{2}+\alpha_{n}\left\langle\nabla h(\bar{x})-\bar{x}, x_{n+1}-\bar{x}\right\rangle
\end{aligned}
$$

for each $n \in \mathbb{N}$. This gives,

$$
\left\|x_{n+1}-\bar{x}\right\|^{2} \leqslant\left(1-\alpha_{n}\left(1-\kappa^{2}\right)\right)\left\|x_{n}-\bar{x}\right\|^{2}+\alpha_{n}\left(1-\kappa^{2}\right)\left(\frac{2}{1-\kappa^{2}}\left\langle\nabla h(\bar{x})-\bar{x}, x_{n+1}-\bar{x}\right\rangle\right)
$$

for each $n \in \mathbb{N}$.

Now, we consider the following possible two cases.

Case 1: Suppose that there exists $n_{0} \in \mathbb{N}$ such that $\left\{\left\|x_{n}-\bar{x}\right\|\right\}$ is monotonically non-increasing. Then, by Lemma 3.4, it follows that $\left\{\left\|x_{n}-\bar{x}\right\|\right\}$ is a convergent sequence. Consider

$$
\begin{aligned}
\left\|x_{n+1}-\bar{x}\right\|^{2}= & \left\|\alpha_{n} \nabla h\left(u_{n}\right)+\left(1-\alpha_{n}\right) J_{\lambda}^{B_{1}}\left(I-\lambda A_{1}\right) u_{n}-\bar{x}\right\|^{2} \\
= & \left\|\alpha_{n}\left(\nabla h\left(u_{n}\right)-\bar{x}\right)+\left(1-\alpha_{n}\right)\left(J_{\lambda}^{B_{1}}\left(I-\lambda A_{1}\right) u_{n}-\bar{x}\right)\right\|^{2} \\
= & \alpha_{n}\left\|\nabla h\left(u_{n}\right)-\bar{x}\right\|^{2}+\left(1-\alpha_{n}\right)\left\|J_{\lambda}^{B_{1}}\left(I-\lambda A_{1}\right) u_{n}-\bar{x}\right\|^{2} \\
& -\alpha_{n}\left(1-\alpha_{n}\right)\left\|\left(\nabla h\left(u_{n}\right)-\bar{x}\right)-\left(J_{\lambda}^{B_{1}}\left(I-\lambda A_{1}\right) u_{n}-\bar{x}\right)\right\|^{2} \\
\leqslant & \alpha_{n}\left\|\nabla h\left(u_{n}\right)-\bar{x}\right\|^{2}+\left(1-\alpha_{n}\right)\left\|u_{n}-\bar{x}\right\|^{2}-\alpha_{n}\left(1-\alpha_{n}\right)\left\|\nabla h\left(u_{n}\right)-J_{\lambda}^{B_{1}}\left(I-\lambda A_{1}\right) u_{n}\right\|^{2} \\
\leqslant & \alpha_{n}\left\|\nabla h\left(u_{n}\right)-\bar{x}\right\|^{2}+\left(1-\alpha_{n}\right)\left\|u_{n}-\bar{x}\right\|^{2}
\end{aligned}
$$

for each $n \in \mathbb{N}$. Then

$$
\left\|u_{n}-\bar{x}\right\|^{2} \geqslant\left\|x_{n+1}-\bar{x}\right\|^{2}-\alpha_{n}\left(\left\|\nabla h\left(u_{n}\right)-\bar{x}\right\|^{2}-\left\|u_{n}-\bar{x}\right\|^{2}\right)
$$

for each $n \in \mathbb{N}$. Next, from (3.2), we have

$$
\begin{aligned}
& \gamma_{n}\left[\left\|\left(I-J_{\lambda}^{B_{2}}\left(I-\lambda A_{2}\right)\right) L x_{n}\right\|^{2}-\gamma_{n}\left(\left\|L^{*}\left(I-J_{\lambda}^{B_{2}}\left(I-\lambda A_{2}\right)\right) L x_{n}\right\|^{2}\right)\right] \\
& \quad \leqslant\left\|x_{n}-\bar{x}\right\|^{2}-\left\|u_{n}-\bar{x}\right\|^{2} \\
& \quad \leqslant\left\|x_{n}-\bar{x}\right\|^{2}-\left\|x_{n+1}-\bar{x}\right\|^{2}+\alpha_{n}\left(\left\|\nabla h\left(u_{n}\right)-\bar{x}\right\|^{2}-\left\|u_{n}-\bar{x}\right\|^{2}\right) \\
& \quad \rightarrow 0,
\end{aligned}
$$


as $n \rightarrow \infty$. By the definition of $\gamma_{n}$, we see that

$$
\begin{array}{r}
\gamma_{n}\left[\left\|\left(I-J_{\lambda}^{B_{2}}\left(I-\lambda A_{2}\right)\right) L x_{n}\right\|^{2}-\gamma_{n}\left(\left\|L^{*}\left(I-J_{\lambda}^{B_{2}}\left(I-\lambda A_{2}\right)\right) L x_{n}\right\|^{2}\right)\right] \\
=\rho_{n}\left(1-\rho_{n}\right) \frac{\left\|\left(I-J_{\lambda}^{B_{2}}\left(I-\lambda A_{2}\right)\right) L x_{n}\right\|^{4}}{\left\|L^{*}\left(I-J_{\lambda}^{B_{2}}\left(I-\lambda A_{2}\right)\right) L x_{n}\right\|^{2}}
\end{array}
$$

for each $n \in \mathbb{N}$. This implies

$$
\rho_{n}\left(1-\rho_{n}\right) \frac{\left\|\left(I-J_{\lambda}^{B_{2}}\left(I-\lambda A_{2}\right)\right) L x_{n}\right\|^{4}}{\left\|L^{*}\left(I-J_{\lambda}^{B_{2}}\left(I-\lambda A_{2}\right)\right) L x_{n}\right\|^{2}} \rightarrow 0,
$$

as $n \rightarrow \infty$. Thus, since $\rho_{n} \in[a, b] \subset(0,1)$, we must have

$$
\lim _{n \rightarrow \infty} \frac{\left\|\left(I-J_{\lambda}^{B_{2}}\left(I-\lambda A_{2}\right)\right) L x_{n}\right\|^{2}}{\left\|L^{*}\left(I-J_{\lambda}^{B_{2}}\left(I-\lambda A_{2}\right)\right) L x_{n}\right\|}=0 .
$$

Next, we observe that the fact $\left\|L^{*}\left(I-J_{\lambda}^{B_{2}}\left(I-\lambda A_{2}\right)\right) L x_{n}\right\| \leqslant\left\|L^{*}\right\|\left\|\left(I-J_{\lambda}^{B_{2}}\left(I-\lambda A_{2}\right)\right) L x_{n}\right\|$ implies

$$
\left\|\left(I-J_{\lambda}^{B_{2}}\left(I-\lambda A_{2}\right)\right) L x_{n}\right\| \leqslant\left\|L^{*}\right\| \frac{\left\|\left(I-J_{\lambda}^{B_{2}}\left(I-\lambda A_{2}\right)\right) L x_{n}\right\|^{2}}{\left\|L^{*}\left(I-J_{\lambda}^{B_{2}}\left(I-\lambda A_{2}\right)\right) L x_{n}\right\|}
$$

for each $n \in \mathbb{N}$. Thus, in view of (3.4), we get

$$
\lim _{n \rightarrow \infty}\left\|\left(I-J_{\lambda}^{B_{2}}\left(I-\lambda A_{2}\right)\right) L x_{n}\right\|=0 .
$$

This forces

$$
\lim _{n \rightarrow \infty}\left\|L^{*}\left(I-J_{\lambda}^{B_{2}}\left(I-\lambda A_{2}\right)\right) L x_{n}\right\|=0 .
$$

At this point, from the definition of $u_{n}$, we see that

$$
\left\|u_{n}-x_{n}\right\|=\left\|x_{n}-\gamma_{n} L^{*}\left(I-J_{\lambda}^{B_{2}}\left(I-\lambda A_{2}\right)\right) L x_{n}-x_{n}\right\| \leqslant \gamma_{n}\left\|L^{*}\left(I-J_{\lambda}^{B_{2}}\left(I-\lambda A_{2}\right)\right) L x_{n}\right\|
$$

for each $n \in \mathbb{N}$. By (3.6), we obtain that

$$
\lim _{n \rightarrow \infty}\left\|u_{n}-x_{n}\right\|=0 \text {. }
$$

Furthermore, it follows from the definition of $x_{n+1}$ that

$$
\begin{aligned}
\left\|x_{n+1}-u_{n}\right\| & =\left\|\alpha_{n} \nabla h\left(u_{n}\right)+\left(1-\alpha_{n}\right) J_{\lambda}^{B_{1}}\left(I-\lambda A_{1}\right) u_{n}-u_{n}\right\| \\
& =\left\|\alpha_{n}\left(\nabla h\left(u_{n}\right)-u_{n}\right)+\left(1-\alpha_{n}\right)\left(J_{\lambda}^{B_{1}}\left(I-\lambda A_{1}\right) u_{n}-u_{n}\right)\right\| \\
& \leqslant \alpha_{n}\left\|\nabla h\left(u_{n}\right)-u_{n}\right\|+\left(1-\alpha_{n}\right)\left\|u_{n}-J_{\lambda}^{B_{1}}\left(I-\lambda A_{1}\right) u_{n}\right\| \\
& \leqslant \alpha_{n}\left\|\nabla h\left(u_{n}\right)-\nabla h(\bar{x})\right\|+\alpha_{n}\left\|\nabla h(\bar{x})-u_{n}\right\|+\left(1-\alpha_{n}\right)\left\|u_{n}-J_{\lambda}^{B_{1}}\left(I-\lambda A_{1}\right) u_{n}\right\| \\
& \leqslant \alpha_{n} k\left\|u_{n}-\bar{x}\right\|+\alpha_{n}\left\|\nabla h(\bar{x})-u_{n}\right\|+\left(1-\alpha_{n}\right)\left\|u_{n}-J_{\lambda}^{B_{1}}\left(I-\lambda A_{1}\right) u_{n}\right\|
\end{aligned}
$$

for each $n \in \mathbb{N}$. By using $\left\|J_{\lambda}^{B_{1}}\left(I-\lambda A_{1}\right) u_{n}-\bar{x}\right\| \geqslant\left\|x_{n+1}-\bar{x}\right\|-\alpha_{n}\left\|\nabla h\left(u_{n}\right)-J_{\lambda}^{B_{1}}\left(I-\lambda A_{1}\right) u_{n}\right\|$ and the nonexpansiveness of $J_{\lambda}^{B_{1}}\left(I-\lambda A_{1}\right)$, we see that

$$
\begin{aligned}
0 & \leqslant\left\|u_{n}-\bar{x}\right\|-\left\|J_{\lambda}^{B_{1}}\left(I-\lambda A_{1}\right) u_{n}-J_{\lambda}^{B_{1}}\left(I-\lambda A_{1}\right) \bar{x}\right\| \\
& =\left\|u_{n}-\bar{x}\right\|-\left\|J_{\lambda}^{B_{1}}\left(I-\lambda A_{1}\right) u_{n}-\bar{x}\right\| \\
& \leqslant\left\|x_{n}-\bar{x}\right\|+\alpha_{n}\left\|\nabla h\left(u_{n}\right)-J_{\lambda}^{B_{1}}\left(I-\lambda A_{1}\right) u_{n}\right\|-\left\|x_{n+1}-\bar{x}\right\|
\end{aligned}
$$


for each $n \in \mathbb{N}$. Subsequently, since $\left\{\left\|x_{n}-\bar{x}\right\|\right\}$ is a convergent sequence, by the conditions (i) and the boundedness of $\left\{u_{n}\right\}$, we obtain that

$$
\left\|u_{n}-\bar{x}\right\|-\left\|J_{\lambda}^{B_{1}}\left(I-\lambda A_{1}\right) u_{n}-J_{\lambda}^{B_{1}}\left(I-\lambda A_{1}\right) \bar{x}\right\| \rightarrow 0,
$$

as $n \rightarrow \infty$. Thus, by the strong nonexpansiveness of $J_{\lambda}^{B_{1}}\left(I-\lambda A_{1}\right)$, we get

$$
\lim _{n \rightarrow \infty}\left\|u_{n}-J_{\lambda}^{B_{1}}\left(I-\lambda A_{1}\right) u_{n}\right\|=0 .
$$

From (3.8), by using the condition (i) and (3.9), we obtain that

$$
\lim _{n \rightarrow \infty}\left\|x_{n+1}-u_{n}\right\|=0,
$$

and this together with (3.7) imply that

$$
\left\|x_{n+1}-x_{n}\right\| \leqslant\left\|x_{n+1}-u_{n}\right\|+\left\|u_{n}-x_{n}\right\| \rightarrow 0,
$$

as $\mathrm{n} \rightarrow \infty$.

Consider, for each $n \in \mathbb{N}$,

$$
\begin{aligned}
\left\|x_{n}-J_{\lambda}^{B_{1}}\left(I-\lambda A_{1}\right) x_{n}\right\| \leqslant & \left\|x_{n}-x_{n+1}\right\|+\left\|x_{n+1}-J_{\lambda}^{B_{1}}\left(I-\lambda A_{1}\right) u_{n}\right\| \\
& +\left\|J_{\lambda}^{B_{1}}\left(I-\lambda A_{1}\right) u_{n}-J_{\lambda}^{B_{1}}\left(I-\lambda A_{1}\right) x_{n}\right\| \\
\leqslant & \left\|x_{n}-x_{n+1}\right\|+\left\|x_{n+1}-J_{\lambda}^{B_{1}}\left(I-\lambda A_{1}\right) u_{n}\right\|+\left\|u_{n}-x_{n}\right\| .
\end{aligned}
$$

In the second term of (3.11), by the definition of $x_{n+1}$, it follows that

$$
\begin{aligned}
\left\|x_{n+1}-J_{\lambda}^{B_{1}}\left(I-\lambda A_{1}\right) u_{n}\right\| & =\left\|\alpha_{n} \nabla h\left(u_{n}\right)+\left(1-\alpha_{n}\right) J_{\lambda}^{B_{1}}\left(I-\lambda A_{1}\right) u_{n}-J_{\lambda}^{B_{1}}\left(I-\lambda A_{1}\right) u_{n}\right\| \\
& =\alpha_{n}\left\|\nabla h\left(u_{n}\right)-J_{\lambda}^{B_{1}}\left(I-\lambda A_{1}\right) u_{n}\right\| \\
& \leqslant \alpha_{n}\left\|\nabla h\left(u_{n}\right)-\nabla h(\bar{x})\right\|+\alpha_{n}\left\|\nabla h(\bar{x})-J_{\lambda}^{B_{1}}\left(I-\lambda A_{1}\right) u_{n}\right\| \\
& \leqslant \alpha_{n} \kappa\left\|u_{n}-\bar{x}\right\|+\alpha_{n}\left\|\nabla h(\bar{x})-J_{\lambda}^{B_{1}}\left(I-\lambda A_{1}\right) u_{n}\right\| \\
& \rightarrow 0
\end{aligned}
$$

as $n \rightarrow \infty$. Substituting (3.7), (3.10), and (3.12) into (3.11), we get

$$
\lim _{n \rightarrow \infty}\left\|x_{n}-J_{\lambda}^{B_{1}}\left(I-\lambda A_{1}\right) x_{n}\right\|=0 .
$$

Next, since $\left\{x_{n}\right\}$ is bounded on $H_{1}$, there exists a subsequence $\left\{x_{n_{i}}\right\}$ of $\left\{x_{n}\right\}$ that converges weakly to $x^{*} \in H_{1}$. We will show that $x^{*} \in\left(A_{1}+B_{1}\right)^{-1} 0$. Consider, for each $i \in \mathbb{N}$,

$$
\begin{aligned}
\left\|\left(I-J_{\lambda}^{B_{1}}\left(I-\lambda A_{1}\right)\right) x^{*}\right\|^{2} \leqslant & \left\langle\left(I-J_{\lambda}^{B_{1}}\left(I-\lambda A_{1}\right)\right) x^{*}, x^{*}-x_{n_{i}}\right\rangle \\
& +\left\langle\left(I-J_{\lambda}^{B_{1}}\left(I-\lambda A_{1}\right)\right) x^{*}, x_{n_{i}}-J_{\lambda}^{B_{1}}\left(I-\lambda A_{1}\right) x_{n_{i}}\right\rangle \\
& +\left\langle\left(I-J_{\lambda}^{B_{1}}\left(I-\lambda A_{1}\right)\right) x^{*}, J_{\lambda}^{B_{1}}\left(I-\lambda A_{1}\right) x_{n_{i}}-J_{\lambda}^{B_{1}}\left(I-\lambda A_{1}\right) x^{*}\right\rangle .
\end{aligned}
$$

Since $\left\{x_{n_{i}}\right\}$ is a subsequence of $\left\{x_{n}\right\}$, so consequence from (3.13) we have

$$
\lim _{i \rightarrow \infty}\left\|x_{n_{i}}-J_{\lambda}^{B_{1}}\left(I-\lambda A_{1}\right) x_{n_{i}}\right\|=0 .
$$

From (3.14), by using (3.15) and together with $x_{n_{i}} \rightarrow x^{*}$, we obtain

$$
\lim _{i \rightarrow \infty}\left\|\left(I-J_{\lambda}^{B_{1}}\left(I-\lambda A_{1}\right)\right) x^{*}\right\|=0 .
$$


Therefore, $x^{*}=J_{\lambda}^{B_{1}}\left(I-\lambda A_{1}\right) x^{*}$ and hence $x^{*} \in\left(A_{1}+B_{1}\right)^{-1} 0$.

Next, we will show that $L x^{*} \in\left(A_{2}+B_{2}\right)^{-1} 0$, that is $x^{*} \in \Gamma$. Similarly, we consider, for each $i \in \mathbb{N}$,

$$
\begin{aligned}
\left\|\left(I-J_{\lambda}^{B_{2}}\left(I-\lambda A_{2}\right)\right) L x^{*}\right\|^{2} \leqslant & \left\langle L x^{*}-J_{\lambda}^{B_{2}}\left(I-\lambda A_{2}\right) L x^{*}, L x^{*}-L u_{n_{i}}\right\rangle \\
& +\left\langle L x^{*}-J_{\lambda}^{B_{2}}\left(I-\lambda A_{2}\right) L x^{*}, L u_{n_{i}}-J_{\lambda}^{B_{2}}\left(I-\lambda A_{2}\right) L u_{n_{i}}\right\rangle \\
& +\left\langle L x^{*}-J_{\lambda}^{B_{2}}\left(I-\lambda A_{2}\right) L x^{*}, J_{\lambda}^{B_{2}}\left(I-\lambda A_{2}\right) L u_{n_{i}}-J_{\lambda}^{B_{2}}\left(I-\lambda A_{2}\right) L x^{*}\right\rangle .
\end{aligned}
$$

To estimate the second term in (3.16), we first consider the following inequality,

$$
\begin{aligned}
\left\|\left(I-J_{\lambda}^{B_{2}}\left(I-\lambda A_{2}\right)\right) L u_{n}\right\| \leqslant & \left\|\left(I-J_{\lambda}^{B_{2}}\left(I-\lambda A_{2}\right)\right) L u_{n}-\left(I-J_{\lambda}^{B_{2}}\left(I-\lambda A_{2}\right)\right) L x_{n}\right\| \\
& +\left\|\left(I-J_{\lambda}^{B_{2}}\left(I-\lambda A_{2}\right)\right) L x_{n}\right\| \\
\leqslant & \left\|L u_{n}-L x_{n}\right\|+\left\|J_{\lambda}^{B_{2}}\left(I-\lambda A_{2}\right) L u_{n}-J_{\lambda}^{B_{2}}\left(I-\lambda A_{2}\right) L x_{n}\right\| \\
& +\left\|\left(I-J_{\lambda}^{B_{2}}\left(I-\lambda A_{2}\right)\right) L x_{n}\right\| \\
\leqslant & 2\|L\|\left\|u_{n}-x_{n}\right\|+\left\|\left(I-J_{\lambda}^{B_{2}}\left(I-\lambda A_{2}\right)\right) L x_{n}\right\|
\end{aligned}
$$

for each $n \in \mathbb{N}$. Then, by (3.5) and (3.7), we have

$$
\lim _{n \rightarrow \infty}\left\|\left(I-J_{\lambda}^{B_{2}}\left(I-\lambda A_{2}\right)\right) L u_{n}\right\|=0 .
$$

Thus, for any subsequence $\left\{u_{n_{i}}\right\}$ of $\left\{u_{n}\right\}$, we also have

$$
\lim _{i \rightarrow \infty}\left\|\left(I-J_{\lambda}^{B_{2}}\left(I-\lambda A_{2}\right)\right) L u_{n_{i}}\right\|=0 .
$$

Moreover, by the linearity and continuity of $L, L x_{n_{i}} \rightarrow L x^{*}$, as $i \rightarrow \infty$ and $\left\|u_{n}-x_{n}\right\| \rightarrow 0$, as $n \rightarrow \infty$, which imply that $L u_{n_{i}} \rightarrow L x^{*}$, as $i \rightarrow \infty$. Hence, from (3.16) we obtain that

$$
\lim _{i \rightarrow \infty}\left\|\left(I-J_{\lambda}^{B_{2}}\left(I-\lambda A_{2}\right)\right) L x^{*}\right\|=0 .
$$

Therefore, $L x^{*}=J_{\lambda}^{B_{2}}\left(I-\lambda A_{2}\right) L x^{*}$, that is $L x^{*} \in\left(A_{2}+B_{2}\right)^{-1} 0$. Consequently, we have $x^{*} \in \Gamma$.

Next, we will show that $\left\{x_{n}\right\}$ converges strongly to $\bar{x}=P_{\Gamma} \nabla h(\bar{x})$. From (3.10) we know that $\| x_{n+1}-$ $x_{n} \| \rightarrow 0$, as $n \rightarrow \infty$. Thus, we can choose a subsequence $\left\{x_{n_{i}+1}\right\}$ of $\left\{x_{n+1}\right\}$, such that

$$
\limsup _{n \rightarrow \infty} \frac{2}{1-\kappa^{2}}\left\langle\nabla h(\bar{x})-\bar{x}, x_{n+1}-\bar{x}\right\rangle=\lim _{i \rightarrow \infty} \frac{2}{1-\kappa^{2}}\left\langle\nabla h(\bar{x})-\bar{x}, x_{n_{i}+1}-\bar{x}\right\rangle .
$$

Since $\left\|x_{n+1}-x_{n}\right\| \rightarrow 0$, as $n \rightarrow \infty$ and $x_{n_{i}} \rightarrow x^{*}$, it follows that $x_{n_{i}+1} \rightarrow x^{*}$. Subsequently, from (3.17) we obtain

$$
\limsup _{n \rightarrow \infty} \frac{2}{1-\kappa^{2}}\left\langle\nabla h(\bar{x})-\bar{x}, x_{n+1}-\bar{x}\right\rangle=\frac{2}{1-\kappa^{2}}\left\langle\nabla h(\bar{x})-\bar{x}, x^{*}-\bar{x}\right\rangle \leqslant 0 .
$$

By using Lemma 2.2 in (3.3), we can conclude that $\left\|x_{n}-\bar{x}\right\| \rightarrow 0$, as $n \rightarrow \infty$. Thus $x_{n} \rightarrow \bar{x}$, as $n \rightarrow \infty$. Since $\left\|u_{n}-x_{n}\right\| \rightarrow 0$, as $n \rightarrow \infty$, so we conclude $u_{n} \rightarrow \bar{x}$, as $n \rightarrow \infty$.

Case 2: If $\left\{\left\|x_{n}-\bar{x}\right\|\right\}$ is not monotonically decreasing sequence. Let us put $\Gamma_{n}=\left\|x_{n}-\bar{x}\right\|, \forall n \in \mathbb{N}$ and let $\tau: \mathbb{N} \rightarrow \mathbb{N}$ be a mapping for all $n \geqslant n_{0}$ (for some $n_{0}$ large enough) by

$$
\tau(n):=\max \left\{k \in \mathbb{N}: k \leqslant n, \quad \Gamma_{k} \leqslant \Gamma_{k+1}\right\} .
$$

Obviously, $\tau$ is a non decreasing sequence, such that $\tau(n) \rightarrow \infty$ as $n \rightarrow \infty$ and

$$
0 \leqslant \Gamma_{\tau(\mathfrak{n})} \leqslant \Gamma_{\tau(\mathfrak{n})+1}, \quad \forall \mathrm{n} \geqslant \mathrm{n}_{0} .
$$


By the similar argument as above in Case 1, we can conclude that

$$
\begin{aligned}
& \lim _{n \rightarrow \infty}\left\|\left(I-J_{\lambda}^{B_{2}}\left(I-\lambda A_{2}\right)\right) L x_{\tau(n)}\right\|=0, \\
& \lim _{n \rightarrow \infty}\left\|L^{*}\left(I-J_{\lambda}^{B_{2}}\left(I-\lambda A_{2}\right)\right) L x_{\tau(n)}\right\|=0, \\
& \lim _{n \rightarrow \infty}\left\|x_{\tau(n)+1}-x_{\tau(n)}\right\|=0,
\end{aligned}
$$

and

$$
\limsup _{n \rightarrow \infty} \frac{2}{1-\kappa^{2}}\left\langle\nabla h(\bar{x})-\bar{x}, x_{\tau(n)+1}-\bar{x}\right\rangle \leqslant 0 .
$$

Since $\left\{x_{\tau(n)}\right\}$ is bounded, we can find a subsequence of $\left\{x_{\tau(n)}\right\}$, still denoted by $\left\{x_{\tau(n)}\right\}$, which converges weakly to $x^{*} \in\left(A_{1}+B_{1}\right)^{-1} 0$ and $L x^{*} \in\left(A_{2}+B_{2}\right)^{-1} 0$. Thus, it follows from (3.3) that

$$
\left\|x_{\tau(n)+1}-\bar{x}\right\|^{2} \leqslant\left(1-\alpha_{\tau(n)}\left(1-\kappa^{2}\right)\right)\left\|x_{\tau(n)}-\bar{x}\right\|^{2}+\alpha_{\tau(n)}\left(1-\kappa^{2}\right)\left(\frac{2}{1-k^{2}}\left\langle\nabla h(\bar{x})-\bar{x}, x_{\tau(n)+1}-\bar{x}\right\rangle\right)
$$

for each $\tau(n) \in \mathbb{N}$. Since $\Gamma_{\tau(n)} \leqslant \Gamma_{\tau(n)+1}$, we obtain that $\left\|\chi_{\tau(n)}-\bar{x}\right\|^{2}-\left\|x_{\tau(n)+1}-\bar{x}\right\|^{2} \leqslant 0$ for each $\tau(n) \in \mathbb{N}$. Thus, from (3.18), we have

$$
\begin{aligned}
\alpha_{\tau(n)}\left(1-\kappa^{2}\right)\left\|x_{\tau(n)}-\bar{x}\right\|^{2} \leqslant & \left\|x_{\tau(n)}-\bar{x}\right\|^{2}-\left\|x_{\tau(n)+1}-\bar{x}\right\|^{2} \\
& +\alpha_{\tau(n)}\left(1-\kappa^{2}\right)\left(\frac{2}{1-\kappa^{2}}\left\langle\nabla h(\bar{x})-\bar{x}, x_{\tau(n)+1}-\bar{x}\right\rangle\right) \\
\leqslant & \alpha_{\tau(n)}\left(1-\kappa^{2}\right)\left(\frac{2}{1-\kappa^{2}}\left\langle\nabla h(\bar{x})-\bar{x}, x_{\tau(n)+1}-\bar{x}\right\rangle\right)
\end{aligned}
$$

for each $\tau(n) \in \mathbb{N}$. Now, we note that $\alpha_{\tau(n)}\left(1-k^{2}\right)>0$, then from (3.19), we get

$$
\limsup _{n \rightarrow \infty}\left\|x_{\tau(n)}-\bar{x}\right\|^{2} \leqslant 0 \text {. }
$$

This implies that

$$
\lim _{n \rightarrow \infty}\left\|x_{\tau(n)}-\bar{x}\right\|^{2}=0
$$

and hence

$$
\lim _{n \rightarrow \infty}\left\|x_{\tau(n)}-\bar{x}\right\|=0 .
$$

By using above $\lim _{n \rightarrow \infty}\left\|x_{\tau(n)+1}-x_{\tau(n)}\right\|=0$ and (3.20), we have that

$$
\left\|x_{\tau(n)+1}-\bar{x}\right\| \leqslant\left\|x_{\tau(n)+1}-x_{\mathcal{\tau}(n)}\right\|+\left\|x_{\tau(n)}-\bar{x}\right\| \rightarrow 0,
$$

as $n \rightarrow \infty$. Furthermore, for $n \geqslant n_{0}$, it is easy to see that $\Gamma_{\tau(n)} \leqslant \Gamma_{\tau(n)+1}$ if $n \neq \tau(n)$ (that is, $\tau(n)<n$ ), because $\Gamma_{j} \geqslant \Gamma_{j+1}$ for $\tau(n)+1 \leqslant j \leqslant n$. As a consequence, we obtain for all $n \geqslant n_{0}$

$$
0 \leqslant \Gamma_{\mathrm{n}} \leqslant \max \left\{\Gamma_{\tau(\mathfrak{n})}, \Gamma_{\tau(\mathfrak{n})+1}\right\}=\Gamma_{\tau(\mathfrak{n})+1}
$$

By using (3.21), we can conclude that $\lim _{n \rightarrow \infty} \Gamma_{n}=0$, that is, $\left\{x_{n}\right\}$ converges strongly to $\bar{x}$. This completes the proof.

\section{Applications}

In this section, we will show some applications of the problem (1.6) via Theorem 3.5.

\subsection{Split variational inequality problem}

Recall that the normal cone to $\mathrm{C}$ at $u \in \mathrm{C}$ is defined as

$$
\mathrm{N}_{\mathrm{C}}(\mathrm{u})=\{z \in \mathrm{H}:\langle z, \mathrm{y}-\mathrm{u}\rangle \leqslant 0, \quad \forall \mathrm{y} \in \mathrm{C}\},
$$

where $\mathrm{C}$ is a nonempty closed convex subset of $\mathrm{H}$. It is well known that $\mathrm{N}_{C}$ is a maximal monotone 
operator. So, in the case $\mathrm{B}:=\mathrm{N}_{\mathrm{C}}: \mathrm{H} \rightarrow 2^{\mathrm{H}}$ we can verify that the problem (1.5) is reduced to the problem of finding $x^{*} \in \mathrm{C}$ such that

$$
\left\langle A x^{*}, x-x^{*}\right\rangle \geqslant 0, \quad \forall x \in C .
$$

Thus, in the case that $\mathrm{B}_{1}:=\mathrm{N}_{\mathrm{C}_{1}}$ and $\mathrm{B}_{2}:=\mathrm{N}_{\mathrm{C}_{2}}$, we get $\mathrm{J}_{\lambda}^{\mathrm{B}_{1}}=: \mathrm{P}_{\mathrm{C}_{1}}$ and $\mathrm{J}_{\lambda}^{\mathrm{B}_{2}}=: \mathrm{P}_{\mathrm{C}_{2}}\left(\mathrm{P}_{\mathrm{C}_{1}}, \mathrm{P}_{\mathrm{C}_{2}}\right.$ are the metric projections onto $C_{1}$ and $C_{2}$, respectively). Subsequently, the problem (1.6) is reduced to the following form of split variational inequality problem:

$$
\text { find } x^{*} \in C_{1} \text { such that }\left\langle A_{1} x^{*}, x-x^{*}\right\rangle \geqslant 0, \quad \forall x \in C_{1}
$$

and such that

$$
\mathrm{L} x^{*} \in \mathrm{C}_{2} \text { solves }\left\langle\mathrm{A}_{2} \mathrm{~L} x^{*}, \mathrm{y}-\mathrm{L} x^{*}\right\rangle \geqslant 0, \quad \forall \mathrm{y} \in \mathrm{C}_{2} .
$$

This problem was studied by many authors, see $[11,18,19]$ for example. Now, we will denote by $\Gamma^{A_{1}, A_{2}}$ for the solution set of problem (4.1) and introduce the following algorithm.

Algorithm 4.1. Let $\left\{\alpha_{n}\right\} \subset(0,1)$ and an initial $x_{1} \in \mathrm{H}_{1}$ be arbitrary, define

$$
\left\{\begin{array}{l}
u_{n}=x_{n}-\gamma_{n} L^{*}\left(I-P_{C_{2}}\left(I-\lambda A_{2}\right)\right) L x_{n}, \\
x_{n+1}=\alpha_{n} \nabla h\left(u_{n}\right)+\left(1-\alpha_{n}\right) P_{C_{1}}\left(I-\lambda A_{1}\right) u_{n}, \quad \forall n \in \mathbb{N},
\end{array}\right.
$$

where the stepsizes sequence $\left\{\gamma_{n}\right\}$ is depend on $\rho_{n} \in[a, b] \subset(0,1)$ by

$$
\gamma_{n}=\left\{\begin{array}{lll}
\rho_{n} \frac{\left\|\left(I-P_{C_{2}}\left(I-\lambda A_{2}\right)\right) L x_{n}\right\|^{2}}{\left\|L *\left(I-P_{C_{2}}\left(I-\lambda A_{2}\right)\right) L x_{n}\right\|^{2}}, & \text { if } \quad L^{*}\left(I-P_{C_{2}}\left(I-\lambda A_{2}\right)\right) L x_{n} \neq 0 \\
\gamma, & \text { if } \text { otherwise }
\end{array}\right.
$$

where $\gamma$ is any nonnegative value.

Subsequently, by applying Theorem 3.5, we obtain the following theorem.

Theorem 4.2. Let $\mathrm{H}_{1}$ and $\mathrm{H}_{2}$ be two real Hilbert spaces and let $\mathrm{C}_{1}$ and $\mathrm{C}_{2}$ be a nonempty closed convex subset of $\mathrm{H}_{1}$ and $\mathrm{H}_{2}$, respectively. Let $\left\{\mathrm{x}_{\mathrm{n}}\right\}$ be generated by Algorithm 4.1. Suppose that the assumptions (A1), (A4), and (A5) hold, $\Gamma^{\mathrm{A}_{1}, \mathrm{~A}_{2}} \neq \emptyset$, and the following control conditions are satisfied:

(i) $\lim _{n \rightarrow \infty} \alpha_{n}=0$;

(ii) $\sum_{n=1}^{\infty} \alpha_{n}=\infty$.

Then, $\left\{u_{n}\right\}$ and $\left\{x_{n}\right\}$ both converge strongly to $\bar{x} \in \Gamma^{A_{1}, A_{2}}$, where $\bar{x}=P_{\Gamma^{A_{1}}, A_{2}} \nabla h(\bar{x})$, which is the optimality condition for the minimization problem

$$
\min _{x \in \Gamma^{A_{1}, A_{2}}} \frac{1}{2}\|x\|^{2}-h(x) .
$$

Proof. It follows immediately from Theorem 3.5 and above setting.

\subsection{Split minimization problem}

We will consider a convex Fréchet differentiable function $f: H \rightarrow R$. Let $C$ be a given closed convex subset of $H$. By setting $A:=\nabla f$ and $B:=N_{C}$, the problem of finding $x^{*} \in(A+B)^{-1} 0$ is equivalent to find a point $x^{*} \in \mathrm{C}$ such that

$$
\left\langle\nabla f\left(x^{*}\right), x-x^{*}\right\rangle \geqslant 0, \quad \forall x \in C .
$$

Note that, (4.2) is equivalent to the following minimization problem: find $x^{*} \in \mathrm{C}$ such that

$$
x^{*} \in \arg \min _{x \in C} f(x) .
$$

Thus, in the case that $A_{1}:=\nabla f, A_{2}:=\nabla g, B_{1}:=N_{C_{1}}$, and $B_{2}:=N_{C_{2}}$, the problem (1.6) is reduced to the 
following split minimization problem:

$$
\text { find } x^{*} \in C_{1} \text { such that } x^{*} \in \arg \min _{x \in C_{1}} f(x) \text {, }
$$

and such that

$$
\mathrm{Lx}^{*} \in \mathrm{C}_{2} \text { solves } \mathrm{L} x^{*} \in \arg \min _{\mathrm{y} \in \mathrm{C}_{2}} \mathrm{~g}(\mathrm{y}) .
$$

We will denote by $\Gamma^{f, g}$ for the solution set of problem (4.3) and introduce the following algorithm.

Algorithm 4.3. Let $\left\{\alpha_{n}\right\} \subset(0,1)$ and an initial $\chi_{1} \in \mathrm{H}_{1}$ be arbitrary, define

$$
\left\{\begin{array}{l}
u_{n}=x_{n}-\gamma_{n} L^{*}\left(I-P_{C_{2}}(I-\lambda \nabla g)\right) L x_{n}, \\
x_{n+1}=\alpha_{n} \nabla h\left(u_{n}\right)+\left(1-\alpha_{n}\right) P_{C_{1}}(I-\lambda \nabla f) u_{n}, \quad \forall n \in \mathbb{N},
\end{array}\right.
$$

where the stepsizes sequence $\left\{\gamma_{n}\right\}$ depends on $\rho_{n} \in[a, b] \subset(0,1)$ by

$$
\gamma_{n}=\left\{\begin{array}{lll}
\rho_{n} \frac{\left\|\left(I-P_{C_{2}}(I-\lambda \nabla g)\right) L x_{n}\right\|^{2}}{\left\|L^{*}\left(I-P_{C_{2}}(I-\lambda \nabla g)\right) L x_{n}\right\|^{2}}, & \text { if } \quad L^{*}\left(I-P_{C_{2}}(I-\lambda \nabla g)\right) L x_{n} \neq 0, \\
\gamma, & \text { if otherwise, }
\end{array}\right.
$$

where $\gamma$ is any nonnegative value.

Then, by applying Theorem 3.5, we obtain the following result.

Theorem 4.4. Let $\mathrm{H}_{1}$ and $\mathrm{H}_{2}$ be two real Hilbert spaces and let $\mathrm{C}_{1}$ and $\mathrm{C}_{2}$ be nonempty closed convex subsets of $\mathrm{H}_{1}$ and $\mathrm{H}_{2}$, respectively. Let $\mathrm{f}: \mathrm{H}_{1} \rightarrow \mathbb{R}$ and $\mathrm{g}: \mathrm{H}_{2} \rightarrow \mathbb{R}$ be convex and Fréchet differentiable, which $\nabla \mathrm{f}$ be $\alpha$-Lipschitz and $\nabla \mathrm{g}$ be $\beta$-Lipschitz, respectively. Let $\left\{x_{n}\right\}$ be generated by Algorithm 4.3. Suppose that the assumptions (A4) and (A5) hold, $\Gamma^{\mathrm{f}, \mathrm{g}} \neq \emptyset$, and the following control conditions are satisfied:

1. $\lim _{n \rightarrow \infty} \alpha_{n}=0$;

2. $\sum_{n=1}^{\infty} \alpha_{n}=\infty$.

Then, $\left\{u_{n}\right\}$ and $\left\{x_{n}\right\}$ both converge strongly to $\bar{x} \in \Gamma^{f, g}$, where $\bar{x}=P_{\Gamma^{f, g}} \nabla h(\bar{x})$, which is the optimality condition for the minimization problem

$$
\min _{x \in \Gamma^{f, g}} \frac{1}{2}\|x\|^{2}-h(x) .
$$

Proof. Note that for a differentiable convex function, such that its gradient is $\alpha$-Lipschitz continuous, then we know that such gradient mapping will be a $\frac{1}{\alpha}$-ism, (see[2]). Thus, the required result can be obtained immediately from Theorem 3.5.

\subsection{Split common fixed point problem}

Let $\mathrm{V}_{1}: \mathrm{H}_{1} \rightarrow \mathrm{H}_{1}$ and $\mathrm{V}_{2}: \mathrm{H}_{2} \rightarrow \mathrm{H}_{2}$ be nonexpansive mappings. Then, by Lemma 2.1 (iii), we know that $A_{1}:=I-V_{1}$ and $A_{2}:=I-V_{2}$ are $\frac{1}{2}$-ism. Furthermore, since $A_{1} x^{*}=0$ if and only if $x^{*} \in F\left(V_{1}\right)$ and $A_{2} x^{*}=0$ if and only if $x^{*} \in F\left(V_{2}\right)$, we see that the problem (1.6) can be reduced to the problem of finding

$$
x^{*} \in \mathrm{F}\left(\mathrm{V}_{1}\right) \quad \text { such that } \quad \mathrm{L} x^{*} \in \mathrm{F}\left(\mathrm{V}_{2}\right) \text {, }
$$

where $\mathrm{L}: \mathrm{H}_{1} \rightarrow \mathrm{H}_{2}$ is a bounded linear operator. This problem is called the split common fixed point problem (SCFP), and was studied by many authors, see [16, 26, 31, 40] for example. We will denote by $\Gamma \mathrm{V}_{1}, \mathrm{~V}_{2}$ for the solution set of problem (4.4) and introduce the following algorithm.

Algorithm 4.5. Let $\left\{\alpha_{n}\right\} \subset(0,1)$ and an initial $\chi_{1} \in \mathrm{H}_{1}$ be arbitrary, define

$$
\left\{\begin{array}{l}
u_{n}=x_{n}-\gamma_{n} \lambda L^{*}\left(I-V_{2}\right) L x_{n}, \\
x_{n+1}=\alpha_{n} \nabla h\left(u_{n}\right)+\left(1-\alpha_{n}\right)\left(I-\lambda\left(I-V_{1}\right)\right) u_{n}, \quad \forall n \in \mathbb{N},
\end{array}\right.
$$

where the stepsizes sequence $\left\{\gamma_{n}\right\}$ is depend on $\rho_{n} \in[a, b] \subset(0,1)$ by 


$$
\gamma_{n}=\left\{\begin{array}{lll}
\rho_{n} \frac{\left\|\left(I-V_{2}\right) L x_{n}\right\|^{2}}{\left\|L^{*}\left(I-V_{2}\right) L x_{n}\right\|^{2}}, & \text { if } & L^{*}\left(I-V_{2}\right) L x_{n} \neq 0 \\
\gamma, & \text { if } & \text { otherwise }
\end{array}\right.
$$

where $\gamma$ is any nonnegative value.

By applying Theorem 3.5, we can obtain the following result.

Theorem 4.6. Let $\mathrm{H}_{1}$ and $\mathrm{H}_{2}$ be two real Hilbert spaces. Let $\mathrm{V}_{1}: \mathrm{H}_{1} \rightarrow \mathrm{H}_{1}$ and $\mathrm{V}_{2}: \mathrm{H}_{2} \rightarrow \mathrm{H}_{2}$ be nonexpansive

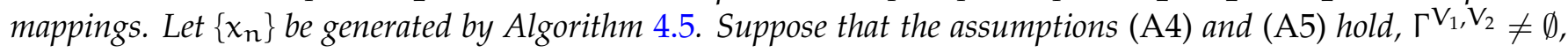
and the following control conditions are satisfied:

(i) $\lim _{n \rightarrow \infty} \alpha_{n}=0$;

(ii) $\sum_{n=1}^{\infty} \alpha_{n}=\infty$.

Then, $\left\{u_{n}\right\}$ and $\left\{x_{n}\right\}$ both converge strongly to $\bar{x} \in \Gamma^{V_{1}}, v_{2}$, where $\bar{x}=P_{\Gamma} v_{1}, v_{2} \nabla h(\bar{x})$, which is the optimality condition for the minimization problem

$$
\min _{x \in \Gamma^{v_{1}, v_{2}}} \frac{1}{2}\|x\|^{2}-h(x)
$$

Proof. Consider $B_{1}:=0$ and $B_{2}:=0$ as zero operators. The required result is followed from the fact that the zero operator is monotone and continuous, hence $B_{1}$ and $B_{2}$ are maximal monotone operators. Moreover, in this case, we see that $J_{\lambda}^{\mathrm{B}_{1}}$ is the identity operator on $\mathrm{H}_{1}$, for each $\lambda>0$ and $\mathrm{J}_{\lambda}^{\mathrm{B}_{2}}$ is the identity operator on $H_{2}$, for each $\lambda>0$. Thus the algorithm (4.5) is defined by setting $A_{1}:=I-V_{1}, A_{2}:=I-V_{2}$ and $\mathrm{B}_{1}:=0, \mathrm{~B}_{2}:=0$.

\section{5. numerical experiments}

In this section, we will show some numerical examples that are related to the main Theorem 3.5 and compare the efficiency between the Algorithm 3.1 and Algorithm (1.7).

Let $n$ be a natural number. Firstly, we recall the some common notations and facts on $\mathbb{R}^{n}$. For $x:=\left(\begin{array}{c}x_{1} \\ x_{2} \\ \vdots \\ x_{n}\end{array}\right) \in \mathbb{R}^{n}$, we will concern with the following notations:

$$
\|x\|_{1}=\left|x_{1}\right|+\left|x_{2}\right|+\cdots+\left|x_{n}\right|
$$

and

$$
\|x\|_{\infty}=\max \left\{\left|x_{1}\right|,\left|x_{2}\right|, \ldots,\left|x_{n}\right|\right\} .
$$

Consider a function $f: \mathbb{R}^{n} \rightarrow \mathbb{R}$, which is defined by

$$
f(x)=\|x\|_{1} \text {, for all } x \in \mathbb{R}^{n} .
$$

We know that $f$ is a convex function and subdifferential of $f$ is

$$
\partial f(x)=\left\{z \in \mathbb{R}^{n}:\langle x, z\rangle=\|x\|_{1},\|z\|_{\infty} \leqslant 1\right\} \text { for all } x \in \mathbb{R}^{n} .
$$

Moreover, since $f$ is convex function, we known that $\partial f(\cdot)$ must be a maximal monotone operator and, for each $\lambda>0$, we have

$$
J_{\lambda}^{\partial f}(x)=\left\{\left(\begin{array}{c}
u_{1} \\
u_{2} \\
\vdots \\
u_{n}
\end{array}\right) \in \mathbb{R}^{n}: u_{i}=x_{i}-\left(\min \left\{\left|x_{i}\right|, \lambda\right\}\right) \operatorname{sgn}\left(x_{i}\right), \text { for } i=1,2, \ldots, n\right\},
$$

where $\operatorname{sgn}(\cdot)$ is denoted for the signum function. Now, we let $H_{1}=\mathbb{R}^{2}$ and $H_{2}=\mathbb{R}^{3}$ be equipped with 
the Euclidean norm. Let $\hat{x}:=\left(\begin{array}{c}3 \\ -4\end{array}\right) \in \mathrm{H}_{1}$ and $\tilde{x}:=\left(\begin{array}{c}3 \\ -2 \\ 3\end{array}\right) \in \mathrm{H}_{2}$ are fixed. We consider 1-ism operators $P_{C}$ and $P_{Q}$, where $C$ and $Q$ are the following convex subset of $H_{1}$ and $H_{2}$, respectively:

$$
\mathrm{C}:=\left\{\mathrm{u} \in \mathrm{H}_{1}:\langle\hat{x}, \mathrm{u}\rangle \leqslant-7\right\}, \quad \mathrm{Q}:=\left\{v \in \mathrm{H}_{2}:\langle\tilde{x}, v\rangle \leqslant-8\right\} .
$$

Let us consider a $3 \times 2$ matrix $L:=\left[\begin{array}{cc}1 & 0 \\ -2 & -1 \\ 1 & 0\end{array}\right]$. We see that $L$ is a bounded linear operator on $H_{1}$ into $\mathrm{H}_{2}$ with $\|\mathrm{L}\|^{2}=6.701562$.

Furthermore, we consider a quadratic function $h: \mathrm{H}_{1} \rightarrow \mathbb{R}$ which is defined by

$$
h(x)=\frac{1}{2} x^{\top} D x-x^{\top} b
$$

where $\mathrm{D}:=\left[\begin{array}{cc}\frac{1}{30} & 0 \\ 0 & \frac{1}{10}\end{array}\right]$, and $\mathrm{b}:=\left[\begin{array}{c}-\frac{29}{30} \\ \frac{3}{2}\end{array}\right]$. We know that $\nabla \mathrm{h}(\mathrm{x}):=\mathrm{Dx}-\mathrm{b}$, and it is a contraction mapping on $\mathrm{H}_{1}$.

Based on above settings, we will present some numerical experiments to show the efficiency of the constructed Algorithm 3.1. That is, we are going to show that the Algorithm 3.1 converges to a point in solution set of the problem:

$$
\text { find } x^{*} \in \mathrm{H}_{1} \text { such that } 0 \in\left(\mathrm{P}_{\mathrm{C}}+\partial \mathrm{f}\right) \mathrm{x}^{*} \text {, }
$$

and such that

$$
\mathrm{Lx}^{*} \in \mathrm{H}_{2} \text { solves } 0 \in\left(\mathrm{P}_{\mathrm{Q}}+\partial \mathrm{g}\right) \mathrm{L} x^{*},
$$

where $f$ and $g$ are defined as in (5.1) on $\mathrm{H}_{1}$ and $\mathrm{H}_{2}$, respectively.

Note that the solution set of the problem (5.2) is $\left\{\left(\begin{array}{c}x \\ -\frac{4 x+1}{3}\end{array}\right) \in \mathrm{H}_{1}: x \geqslant \frac{1}{2}\right\}$. In the experiment, we consider the stopping criterion by $\frac{\left\|x_{n+1}-x_{n}\right\|}{\left\|x_{n+1}\right\|} \leqslant 1.0 e^{-06}$.

In the case that $\lambda=1, \rho_{n}=0.5$. We consider $\alpha_{n}$ in the following different three cases, that are $\alpha_{n}=$ $0.1,0.5,0.9$. We may suggest that, as the presented result in Figure 1, the larger stepsize of parameters $\alpha_{n}$ should provide a more faster convergence. On the other hand, we would like to inform that the changing of $\lambda$ and $\gamma_{n}$ will not make the significant effect to the convergence rate of the Algorithm 3.1.

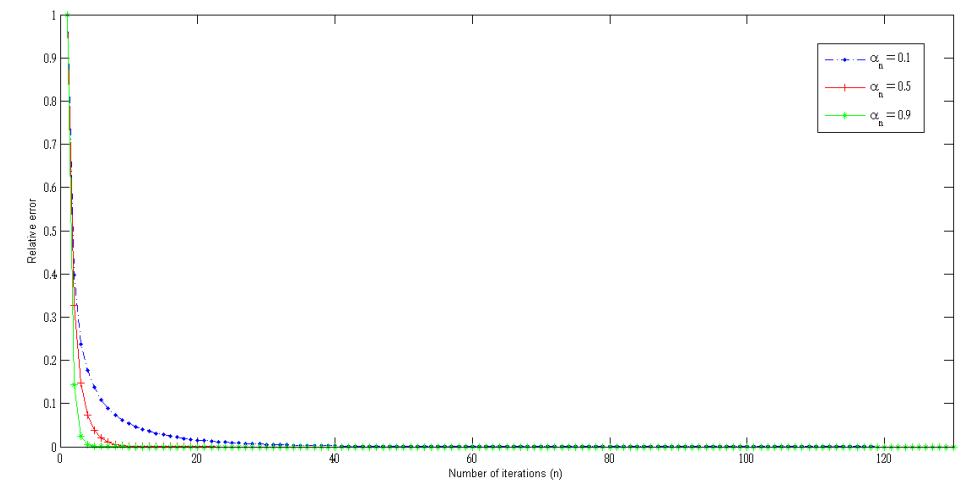

Figure 1: Behavior of the relative error of $x_{n}$ with different $\alpha_{n}$ at the initial vector $(0,0)^{\top}$. 
Next, we consider the Algorithm (1.7) with five cases of parameters $\lambda \in(0,2)$ and $\gamma \in\left(0, \frac{1}{\|\mathrm{~L}\|^{2}}\right)$. The results are showed in Table 1 . Obviously, the choices $\lambda=1.9$ and $\gamma=\frac{0.9}{\|L\|^{2}}$ provide the better convergence rate than other choices.

Finally, in Table 2, we compare the results between the Algorithm 3.1 and Algorithm (1.7) by the initial points $\left(\begin{array}{l}0 \\ 0\end{array}\right),\left(\begin{array}{l}1 \\ 1\end{array}\right),\left(\begin{array}{c}-1 \\ 1\end{array}\right),\left(\begin{array}{c}1 \\ -1\end{array}\right)$, and $\left(\begin{array}{l}-1 \\ -1\end{array}\right)$. In the Algorithm 3.1, we choose $\lambda=1, \rho_{n}=0.5$ and consider different two cases of $\alpha_{n}$, that are $\alpha_{n}=0.1,0.9$. In the Algorithm (1.7), we choose $\lambda=1.9$ and $\gamma=\frac{0.9}{\|L\|^{2}}$. One can see that, the Algorithm 3.1 shows the better convergence rate in almost all the considered cases.

Table 1: Influence of the stepsize parameters $\lambda$ and $\gamma$ of the Algorithm (1.7) for different initial points.

\begin{tabular}{|c|c|c|c|c|c|c|c|c|c|c|}
\hline \multirow{2}{*}{$\begin{array}{l}\text { Case } \rightarrow \\
\text { \#Initial point } \downarrow\end{array}$} & \multicolumn{2}{|c|}{$\lambda=1, \gamma=\frac{0.5}{\|\mathrm{~L}\|^{2}}$} & \multicolumn{2}{|c|}{$\lambda=0.1, \gamma=\frac{0.1}{\|\mathrm{~L}\|^{2}}$} & \multicolumn{2}{|c|}{$\lambda=0.1, \gamma=\frac{0.9}{\|\mathrm{~L}\|^{2}}$} & \multicolumn{2}{|c|}{$\lambda=1.9, \gamma=\frac{0.1}{\|\mathrm{~L}\|^{2}}$} & \multirow{2}{*}{\multicolumn{2}{|c|}{$\lambda=1.9, \gamma=\frac{0.9}{\|\mathrm{~L}\|^{2}} \underset{\text { Sol }}{\text { Iters }}$}} \\
\hline & Iters & Sol & Iters & Sol & Iters & Sol & Iters & Sol & & \\
\hline$(0,0)^{\top}$ & 766 & $\left(\begin{array}{c}0.499945 \\
-0.999926\end{array}\right)$ & 4,823 & $\left(\begin{array}{c}0.499596 \\
-0.999371\end{array}\right)$ & 2,092 & $\left(\begin{array}{c}0.499869 \\
-0.999752\end{array}\right)$ & 3,152 & $\left(\begin{array}{c}0.499716 \\
-0.999627\end{array}\right)$ & 476 & $\left(\begin{array}{c}0.499964 \\
-0.999959\end{array}\right)$ \\
\hline$(-1,1)^{\top}$ & 767 & $\left(\begin{array}{c}0.499945 \\
-0.999926\end{array}\right)$ & 4,828 & $\left(\begin{array}{c}0.499596 \\
-0.999371\end{array}\right)$ & 2,097 & $\left(\begin{array}{c}0.499869 \\
-0.999752\end{array}\right)$ & 3,153 & $\left(\begin{array}{c}0.499716 \\
-0.999627\end{array}\right)$ & 477 & $\left(\begin{array}{c}0.499964 \\
-0.999959\end{array}\right)$ \\
\hline$(-1,-1)^{\top}$ & 749 & $\left(\begin{array}{c}0.499945 \\
-0.999927\end{array}\right)$ & 4,297 & $\left(\begin{array}{c}0.499596 \\
-0.999371\end{array}\right)$ & 1,875 & $\left(\begin{array}{c}0.499868 \\
-0.999751\end{array}\right)$ & 2,823 & $\left(\begin{array}{c}0.499716 \\
-0.999626\end{array}\right)$ & 458 & $\left(\begin{array}{c}0.499964 \\
-0.999959\end{array}\right)$ \\
\hline
\end{tabular}

Table 2: Numerical comparison between the Algorithm $3.1\left(\alpha_{n}=0.1,0.9\right)$ and the Algorithm (1.7) for different initial vectors.

\begin{tabular}{|c|c|c|c|c|c|c|c|c|c|}
\hline \multirow{2}{*}{$\begin{array}{l}\text { Algorithm } \rightarrow \\
\text { \#Initial point } \downarrow\end{array}$} & \multicolumn{6}{|c|}{ Algorithm 3.1} & \multicolumn{3}{|c|}{ Algorithm (1.7) } \\
\hline & Iters & $\begin{array}{r}\alpha_{n}=0.1 \\
\text { Time(Sec) }\end{array}$ & Sol & Iters & $\begin{array}{c}\alpha_{n}=0.9 \\
\text { Time(Sec) }\end{array}$ & Sol & Iters & Time(Sec) & Sol \\
\hline$(0,0)^{\top}$ & 119 & 0.032609 & $\left(\begin{array}{c}0.999989 \\
-1.666651\end{array}\right)$ & 10 & 0.020138 & $\left(\begin{array}{c}1.000000 \\
-1.666666\end{array}\right)$ & 476 & 0.068803 & $\left(\begin{array}{c}0.499964 \\
-0.999959\end{array}\right)$ \\
\hline$(1,1)^{\top}$ & 120 & 0.032707 & $\left(\begin{array}{c}1.000000 \\
-1.666653\end{array}\right)$ & 10 & 0.020255 & $\left(\begin{array}{c}1.000000 \\
-1.666666\end{array}\right)$ & 467 & 0.070894 & $\left(\begin{array}{c}0.499964 \\
-0.999959\end{array}\right)$ \\
\hline$(-1,1)^{\top}$ & 120 & 0.031387 & $\left(\begin{array}{c}1.000000 \\
-1.666653\end{array}\right)$ & 10 & 0.020071 & $\left(\begin{array}{c}1.000000 \\
-1.666666\end{array}\right)$ & 477 & 0.069136 & $\left(\begin{array}{c}0.499964 \\
-0.999959\end{array}\right)$ \\
\hline$(1,-1)^{\top}$ & 107 & 0.030091 & $\left(\begin{array}{c}1.000000 \\
-1.666652\end{array}\right)$ & 10 & 0.020207 & $\left(\begin{array}{c}1.000000 \\
-1.666666\end{array}\right)$ & 16 & 0.024981 & $\left(\begin{array}{c}0.681172 \\
-1.241563\end{array}\right)$ \\
\hline$(-1,-1)^{\top}$ & 118 & 0.031585 & $\left(\begin{array}{c}1.000000 \\
-1.666652\end{array}\right)$ & 10 & 0.019995 & $\left(\begin{array}{c}1.000000 \\
-1.666666\end{array}\right)$ & 458 & 0.067197 & $\left(\begin{array}{c}0.499964 \\
-0.999959\end{array}\right)$ \\
\hline
\end{tabular}

\section{Concluding remarks}

In this work, we are focusing on the split null point problem when the considered problem is arose in the form of sum of monotone operators. We notice that the problem (1.6), which is the main considered problem in this work, can be written in the form of problem (1.4). However, in order to find a solution of (1.6) via the algorithms that were used for solving problem (1.4) may cause the expensive computations in the practical applications. An another extending concept of problem (1.4) is the consideration of difference of two maximal monotone operators, instead of sum of two maximal monotone operators. It is worth to point out that this approach may lead to the (split) non-monotone inclusion problem types, and it should be studied in the future research.

\section{Acknowledgment}

This work is supported by the Thailand Research Fund under the project RSA5880028 and Naresuan University. 


\section{References}

[1] J.-B. Baillon, R. E. Bruck, S. Reich, On the asymptotic behavior of nonexpansive mappings and semigroups in Banach spaces, Houston J. Math., 4 (1978), 1-9. 2

[2] J.-B. Baillon, G. Haddad, Quelques propriétés des opérateurs angle-bornés et n-cycliquement monotones, Israel J. Math., 26 (1977), 137-150. 4.2

[3] H. H. Bauschke, P. L. Combettes, Convex analysis and monotone operator theory in Hilbert spaces, CMS Books in Mathematics/Ouvrages de Mathmatiques de la SMC, Springer, New York, (2011). 1

[4] O. A. Boikanyo, The viscosity approximation forward-backward splitting method for zeros of the sum of monotone operators, Abstr. Appl. Anal., 2016 (2016), 10 pages. 1, 2, 2.1, 2

[5] R. E. Bruck, S. Reich, Nonexpansive projections and resolvents of accretive operators in Banach spaces, Houston J. Math., 3 (1977), 459-470. 1

[6] C. Byrne, Iterative oblique projection onto convex sets and the split feasibility problem, Inverse Problems, 18 (2002), 441-453. 1

[7] C. Byrne, Y. Censor, A. Gibali, S. Reich, Weak and strong convergence of algorithms for the split common null point problem, J. Nonlinear Convex Analysis, 13 (2012), 759-775. 1, 1

[8] A. Cegielski, Iterative methods for fixed point problems in Hilbert Spaces, Lecture Notes in Mathematics, Springer, Heidelberg, (2012). 2, 2

[9] Y. Censor, T. Bortfeld, B. Martin, A. Trofimov, A unified approach for inversion problems in intensity-modulated radiation therapy, Phys. Med. Biol., 51 (2006), 2353-2365. 1

[10] Y. Censor, T. Elfving, A multiprojection algorithm using Bregman projections in product space, Numer. Algorithms, 8 (1994), 221-239. 1

[11] Y. Censor, A. Gibali, S. Reich, Algorithms for the split variational inequality problem, Numer. Algorithms, 59 (2012), 301-323. 4.1

[12] S. S. Change, L. Wang, Y. Zhao, On a class of split equality fixed point problems in Hilbert spaces, J. Nonlinear Var. Anal., 1 (2017), 201-212. 1

[13] S. Y. Cho, B. A. Bin Dehaish, X. Qin, Weak convergence of a splitting algorithm in Hilbert spaces, J. Appl. Anal. Comput., 7 (2017), 427-438.

[14] S. Y. Cho, X. Qin, L. Wang, Strong convergence of a splitting algorithm for treating monotone operators, Fixed Point Theory Appl., 2014 (2014), 15 pages.

[15] P. Cholamjiak, W. Cholamjiak, S. Suantai, A modified regularization method for finding zeros of monotone operators in Hilbert spaces, J. Inequal. Appl., 2015 (2015), 10 pages. 1

[16] H. Cui, F. Wang, Iterative methods for the split common fixed point problem in Hilbert spaces, Fixed Point Theory Appl., 2014 (2014), 8 pages. 4.3

[17] J. Eckstein, D. P. Bertsckas, On the Douglas-Rachford splitting method and the proximal point algorithm for maximal monotone operators, Math. Programming, 55 (1992), 293-318. 1

[18] M. Eslamian, S. Saejung, J. Vahidi, Common solutions of a system of variational inequality problems, Politehn. Univ. Bucharest Sci. Bull. Ser. A Appl. Math. Phys., 77 (2015), 55-62. 4.1

[19] H. He, C. Ling, H.-K. Xu, A relaxed projection method for split variational inequalities, J. Optim. Theory Appl., 166 (2015), 213-233. 4.1

[20] L. S. Liu, Ishikawa and Mann iterative process with errors for nonlinear strongly accretive mappings in Banach spaces, J. Math. Anal. Appl., 194 (1995), 114-125. 2.2

[21] G. López, V. Martín-Márquez, F. Wang, H.-K. Xu, Solving the split feasibility problem without prior knowledge of matrix norms, Inverse Problems, 28 (2012), 18 pages. 1, 1

[22] G. Marino, H.-K. Xu, Convergence of generalized proximal point algorithm, Commun. Pure Appl. Anal., 3 (2004), 791-808. 1

[23] G. Marino, H.-K. Xu, A general iterative method for nonexpansive mappings in Hilbert spaces, J. Math. Anal. Appl., 318 (2006), 43-52. 3.2

[24] B. Martinet, Régularisation d'inéquations variationnelles par approximations successives, Rev. Franaise Informat. Recherche Opérationnelle, 4 (1970), 154-158. 1

[25] A. Moudafi, Viscosity approximation methods for fixed-points problems, J. Math. Anal. Appl., 241 (2000), 46-55. 3.2

[26] A. Moudafi, A note on the split common fixed-point problem for quasi-nonexpansive operators, Nonlinear Anal., 74 (2011), 4083-4087. 4.3

[27] A. Moudafi, Split monotone variational inclusions, J. Optim. Theory Appl., 150 (2011), 275-283. 1, 1

[28] A. Moudafi, M. Théra, Finding a zero of the sum of two maximal monotone operators, J. Optim. Theory Appl., 94 (1997), 425-448. 1

[29] X. Qin, S. Y. Cho, L. Wang, A regularization method for treating zero points of the sum of two monotone operators, Fixed Point Theory Appl., 2014 (2014), 10 pages. 1

[30] X. Qin, J.-C. Yao, Projection splitting algorithms for nonself operators, J. Nonlinear Convex Anal., 18 (2017), $925-935$. 1

[31] T. Shimizu, W. Takahashi, Strong convergence to common fixed points of families of nonexpansive mappings, J. Math. Anal. Appl., 211 (1997), 71-83. 4.3 
[32] M. Suwannaprapa, N. Petrot, S. Suantai, Weak convergence theorems for split feasibility problems on zeros of the sum of monotone operators and fixed point sets in Hilbert spaces, Fixed Point Theory Appl., 2017 (2017), 17 pages. 1

[33] W. Takahashi, Introduction to nonlinear and convex analysis, Yokohama Publishers, Yokohama, (2009). 2

[34] W. Takahashi, M. Toyoda, Weak convergence theorems for nonexpansive mappings and monotone mappings, J. Optim. Theory Appl., 118 (2003), 417-428. 2

[35] W. Takahashi, H.-K. Xu, J.-C. Yao, Iterative methods for generalized split feasibility problems in Hilbert spaces, Set-Valued Var. Anal., 23 (2015), 205-221. 2

[36] P. Tseng, A modified forward-backward splitting method for maximal monotone mappings, SIAM J. Control Optim., 38 (2000), 431-446. 1

[37] H.-K. Xu, Iterative algorithms for nonlinear operators, J. London Math. Soc., 66 (2002), 240-256. 1, 2.2

[38] H.-K. Xu, Averaged mappings and the gradient-projection algorithm, J. Optim. Theory Appl., 150 (2011), 360-378. 2, 2.1

[39] Y. Yao, M. A. Noor, On convergence criteria of generalized proximal point algorithms, J. Comput. Appl. Math., 217 (2008), 46-55. 1

[40] J. Zhao, S. He, Strong convergence of the viscosity approximation process for the split common fixed-point problem of quasi-nonexpansive mappings, J. Appl. Math., 2012 (2012), 12 pages. 4.3 